\title{
The spatial epidemiology and clinical features of reported cases of La Crosse Virus infection in West Virginia from 2003 to 2007
}

\author{
Andrew D Haddow ${ }^{1,2^{*}}$, Danae Bixler ${ }^{3}$, Agricola Odoi $^{4}$
}

\begin{abstract}
Background: La Crosse virus (LACV) is a major cause of pediatric encephalitis in the United States. Since the mid1980s, the number of reported cases of LACV infection in West Virginia has continued to rise and the state currently reports the most cases in the United States. The purpose of this study was to investigate and describe the spatial epidemiology and clinical presentation of LACV infection cases reported in West Virginia, as well as to provide a description of the environmental conditions present at the residences of the LACV infection cases.

Methods: Descriptive and spatial analyses were performed on LACV infection cases reported to the West Virginia Department of Health from 2003 to 2007. Clinical and environmental variables were available for 96 cases and residence data were available for 68 of these cases. Spatial analyses using the global Moran's I and Kulldorff's spatial scan statistic were performed using the population 15 years and younger at both the county and census tract levels to identify those geographic areas at the highest risk of infection.
\end{abstract}

Results: Two statistically significant $(p<0.05)$ high-risk clusters, involving six counties, were detected at the county level. At the census tract level, one statistically significant high-risk cluster involving 41 census tracts spanning over six counties was identified. The county level cumulative incidence for those counties in the primary high-risk cluster ranged from 100.0 to 189.0 cases per 100,000 persons (median 189.0) and the census tract level cumulative incidence for those counties in the high-risk cluster ranged from 61.7 to 505.9 cases per 100,000 persons (median 99.0). The counties and census tracts within high-risk clusters had a relative risk four to nine times higher when compared to those areas not contained within high-risk clusters. The majority of LACV infection cases were reported during the summer months in children 15 years and younger. Fever, vomiting, photophobia, and nausea were the most commonly reported signs and symptoms. A case fatality rate (CFR) of 3.1\% was observed. Wooded areas and containers were present at the majority of case residences.

Conclusions: The cumulative incidences of LACV infection from 2003 to 2007 were considerably higher than previously reported for West Virginia, and statistically significant high-risk clusters for LACV infection were detected at both the county and census tract levels. The finding of a high CFR and the identification of those areas at highest risk for infection will be useful for guiding future research and intervention efforts.

\section{Background}

La Crosse virus (LACV), a member of the genus Orthobunyavirus, family Bunyaviridae, was first isolated from the brain of a pediatric patient who died of encephalitis in 1964 [1]. Since that time LACV has been recognized as one of the most common causes of pediatric arboviral

\footnotetext{
* Correspondence: adhaddow@gmail.com

'The University of Tennessee, Department of Entomology \& Plant Pathology, Knoxville, TN 37996-4560, USA

Full list of author information is available at the end of the article
}

encephalitis in the United States [2,3], with the majority of cases being reported in children under 15 years of age [4-8]. There are an average of 79 reported cases per year nationally [8], however the true incidence of LACV infections remains unknown, as most are undiagnosed and/or underreported $[8,9]$. The virus is transmitted to humans through the bite of infective mosquitoes, the primary vector being the eastern tree-hole mosquito, Aedes triseriatus [10], though two invasive species, the Asian tiger mosquito, Ae. albopictus, and the Asian

\section{Biomed Central}


bush mosquito, Ae. japonicus, have been incriminated as possible secondary or bridge vectors and both species are known to feed on humans [11-16]. La Crosse virus is maintained in a complex cycle involving both horizontal and vertical transmission in mosquitoes, as well as amplification in sciurid hosts [17-21], and is typically associated with areas of dense vegetation and/or stands of hardwood trees where the presence of vector species overlaps that of the amplification hosts [22-24]. The traditionally reported focus of virus transmission to humans has been the upper-Midwestern United States $[2,4,5]$, but more recently LACV has been considered a major cause of pediatric encephalitic disease in West Virginia. Notably, West Virginia has led the nation in the number of annually reported cases since 1994 $[8,25,26]$ (CDC unpublished).

From 1964 to 1986, there were 15 reported cases of LACV infection in West Virginia, comprising $0.9 \%$ of the nationally reported cases. Then in 1987, a pediatric referral center located in Charleston, West Virginia reported several cases of LACV infection. These findings led to active hospital-based surveillance for LACV infection cases in 15 West Virginian counties, ultimately resulting in the detection of 19 additional cases [27]. This case series marked a substantial increase in the number of reported LACV infection cases in the state and led to increased surveillance and research efforts. Following this outbreak, from 1987 to 2007, West Virginia reported 555 cases of La Crosse encephalitis to the Centers for Disease Control and Prevention (CDC), accounting for $31.4 \%$ of the total cases reported in the United States (CDC, unpublished data). A matched case-control study performed following the 1987 outbreak of LACV in West Virginia found a slight increase in disease risk for an increased time spent outdoors, the non-use of insect repellent, the non-use of air conditioning, a lack of screened windows and the non-use of protective clothing, while the presence of tree holes (natural water receptacles) near a residence was found to significantly increase the risk of virus transmission [28].

We conducted spatial and descriptive analyses of cases of LACV infection reported in West Virginia from 2003 to 2007 to determine those geographic areas at the highest risk for human infection and to assess both the clinical presentation of cases and the environmental conditions present at case residences.

\section{Methods}

\section{Case data}

Probable and confirmed LACV infection case data were collected through a passive surveillance system from 2003 to 2007, by the West Virginia Department of Health and Human Resources. Clinical data were available for 96 cases ranging in age from 0.4 years to 54.0 years (78 confirmed and 18 probable cases). Of these cases, 81 were 15 years or younger, of which 68 had data available on the location of their primary residence. The location of the primary residence for each case was determined by a geographic positioning system (GPS) reading taken at each case residence by the West Virginia Department of Health and Human Resources. Personal identifiers of cases were deleted before the data were released for the study. This research was exempted from the Institutional Review Board (IRB) review and certification under section 45 CFR 46.101(b) item 4 of the University of Tennessee's categories for exempted review involving the use of human subjects following a review by the Departmental Review Committee. The above item states that a research study may be exempt from IRB review if the research involves "the collection of or study of existing data, documents, records, pathological specimens, if these sources are publicly available or if the information is recorded by the investigator in such a way that subjects cannot be identified, directly or through identifiers linked to the subjects."

\section{Case definition}

Confirmed cases of LACV infection are required to meet both the clinical and laboratory requirements set by the CDC case definition for neuroinvasive domestic arboviral diseases [29]. This definition is reprinted below:

In the absence of a more likely clinical explanation as documented by a physician, confirmed cases must meet all of the following criteria:

\section{Clinical criteria}

1) Fever, AND 2) Acutely altered mental status, or other acute signs of central or peripheral neurologic dysfunction, or pleocytosis associated with illness clinically compatible with meningitis,

AND

\section{Laboratory criteria}

3) A four-fold or greater change in virus-specific serum antibody titer, or isolation of virus from or demonstration of specific viral antigen or genomic sequences in tissue, blood, CSF, or other body fluid, or virus-specific immunoglobulin $\mathrm{M}$ (IgM) antibodies demonstrated in CSF by antibody-capture enzyme immunoassay (EIA), or virus-specific IgM antibodies demonstrated in serum by antibody-capture EIA and confirmed by demonstration of virus-specific serum immunoglobulin G (IgG) antibodies.

\section{Probable case criteria}

Cases that met the clinical definition and had stable (less than or equal to a two-fold change) but elevated titer of virus-specific serum antibodies, or virus-specific serum IgM antibodies detected by antibody capture EIA 
but with no available results of a confirmatory test for virus-specific serum IgG antibodies in the same or a later specimen, are deemed probable.

\section{Population, geographic, and environmental data}

As the majority of LACV infections are pediatric [2,6,7], it was deemed appropriate to use the population 15 years and younger for the calculation of cumulative incidence for this study. The decennial 2000 United States Census was used to calculate the population 15 years and younger for each county and census tract in West Virginia. Counties are administrative and statistical subdivisions of a state. Census tracts are subdivisions of a county typically containing between 2,500 and 8,000 persons and are relatively homogeneous with respect to population characteristics, economic status and living conditions. Geographic boundary files were downloaded from the United States Census, TIGER, Geodatabase [30], and were used for all cartographic displays. Environmental officers of the West Virginia Department of Health and Human Resources conducted environmental assessments at the primary residence of both probable and confirmed cases' of LACV infection during the study period.

\section{Statistical and geographic analyses}

Cumulative incidences were calculated at both the county and the census tract levels, and spatial analyses were performed on 68 cases 15 years and younger for which location data were available. Cumulative incidences were calculated for all counties in the study area $(\mathrm{n}=55)$ and for counties reporting cases $(\mathrm{n}=18)$, as well as for all census tracts in the study area $(n=466)$ and for census tracts reporting cases $(n=50)$. Cumulative incidence was expressed as the number of cases per 100,000 persons for the study period.

Descriptive analyses and the calculation of cumulative incidences were performed using STATA 10.0 [31]. The non-homogeneity of variances and resulting autocorrelation were adjusted for by smoothing the risk using spatial empirical Bayesian (SEB) smoothing [8,32-36]. This technique was implemented in GeoDa [37] using inverse distance spatial weights.

\section{Detection of Spatial clusters}

The global Moran's I, implemented in GeoDa [37], was used to assess the presence of significant spatial autocorrelation of the unsmoothed cumulative incidences. Detection of spatial clusters of LACV infection were performed using Kulldorff's spatial scan statistic [38], and implemented in SaTScan [39]. A Poisson probability model was used to scan for geographical areas (counties and census tracts) with statistically significant high rates of LACV infections. A maximum spatial cluster size of
$10 \%$ of the population 15 years and younger was used. For statistical inference, 9999 Monte Carlo replications were performed. The null hypothesis of no clusters was rejected when the simulated $\mathrm{p} \leq 0.05$. Cartographic displays were made using ArcView GIS 9.2 [40].

\section{Results}

\section{Spatial analyses}

The highest cumulative incidences for LACV infection cases were observed at the census tract level (Table 1). The cumulative incidence for counties reporting cases ranged from 7.2 to 166.8 per 100,000 persons (median 32.1 ), and from 42.4 to 505.9 per 100,000 persons (median 150.0) for those census tracts reporting cases.

The highest unsmoothed risks were observed in the south-central region of the state at both the county and census tract levels (Figure 1). Visually, the spatial patterns of SEB smoothed risks at the county and census tract levels were more evident and followed patterns similar to those of the unsmoothed risks (Figure 1), though only some of the census tracts within the highrisk counties had high-risks of infection.

Statistically significant global clustering was detected at the county and census tracts with global Moran's I values of $0.4986(\mathrm{p}=0.0001)$ and $0.2935(\mathrm{p}=0.0001)$, respectively. Similarly, statistically significant local clusters $(\mathrm{p}<0.05)$ of high-risk were detected at both the county and the census tract levels (Figure 2). At the county level, two statistically significant high-risk clusters were identified. The primary statistically significant ( $\mathrm{p}=0.0001)$ high-risk cluster consisted of three counties (Fayette, Raleigh, Nicholas). This cluster had a cumulative incidence ranging from 100 to 189 cases per 100,000 persons (median 111) and a relative risk of 9.2 implying that the risk of LACV infection was 9.2 times higher in these three counties than in those counties in the rest of the state of West Virginia. A secondary statistically significant $(\mathrm{p}=0.0012)$ highrisk cluster was identified and included Wyoming, McDowell and Mercer counties. This cluster had a cumulative incidence ranging from 19 to 111 cases per 100,000 persons (median 40) and a relative risk of 4.3 (Figure 2) implying that the risk of LACV infection in this cluster was 4.3 times higher than that of those counties in the rest of the state. At the census tract level, one statistically significant $(\mathrm{p}=0.0001)$ high-risk cluster, consisting of 41 census tracts, was identified. This cluster had a cumulative incidence ranging from 61 to 506 cases per 100,000 persons (median 99.0) and a relative risk of 6.2 , implying that the risk of infection was 6.2 times higher than those census tracts not contained in the cluster. These census tracts were located in the following counties: Clay (3 census tracts), Fayette ( 8 census tracts), Mercer (14 census tracts), 
Table 1 Comparisons of the Cumulative Incidence of Reported La Crosse Virus Infection Cases in the Population 15 Years and Younger for West Virginia County and Census Tracts, 2003 to 2007

\begin{tabular}{clccc}
\hline & & & \multicolumn{2}{c}{ Cumulative incidence per 100,000 persons } \\
\cline { 3 - 4 } Geographic Risk Level & Obs. & Median & Range \\
\hline \multirow{2}{*}{ Entire Study Area } & County & 55 & 0.0 & $0.0-166.8$ \\
& Census tract & 466 & 0.0 & $0.0-505.9$ \\
Reporting Cases & County & 18 & 32.1 & $7.2-166.8$ \\
& Census tract & 50 & 150.0 & $42.4-505.9$ \\
High-Risk Clusters & County & 4 & 83.2 & $40.2-166.8$ \\
& Census tract & 30 & 156.5 & $61.7-505.9$ \\
\hline
\end{tabular}

Obs. = Observations, and refer to the number of counties or census tracts used for each analysis.

Raleigh (13 census tracts), Nicholas (1 census tract), and Summers (2 census tracts) (Figure 2).

\section{Clinical features}

Cases of LACV infection ranged in age from 0.4 to 54.0 years, with a mean age of 10.8 (SD 10.3) years, of which $84.4 \%$ occurred in children 15 years and younger (Table 2).
The vast majority of cases presented during July (28.1\%), August (37.5\%), and September (16.6\%) in the southern region of the state. The most commonly reported signs and symptoms in cases were fever $(76.0 \%)$, vomiting (75.0\%), photophobia (52.1\%), and nausea (50.0\%) (Table $2)$. Other reported signs and symptoms included weakness $(41.6 \%)$, stiff neck and confusion (33.3\%), seizures

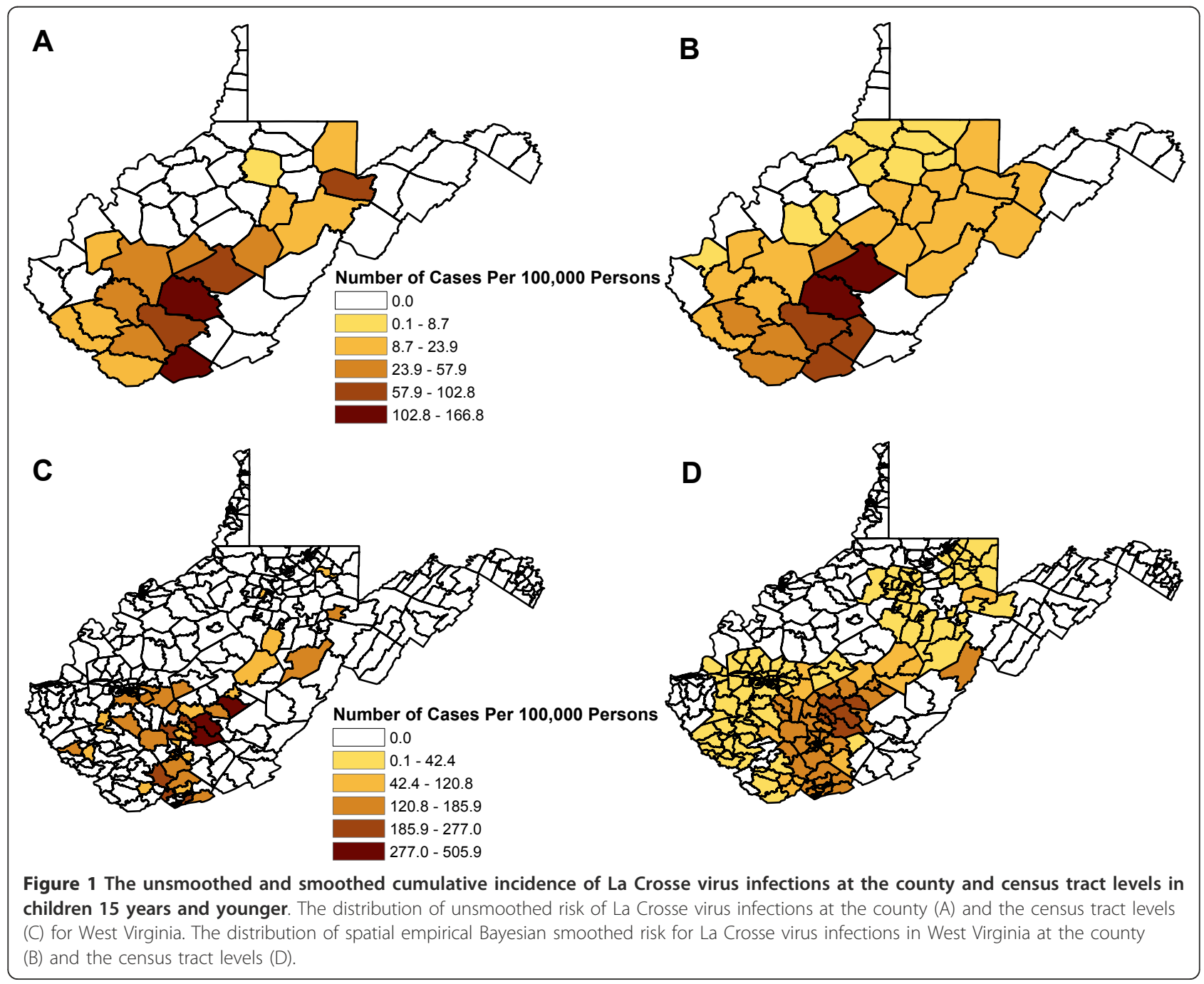



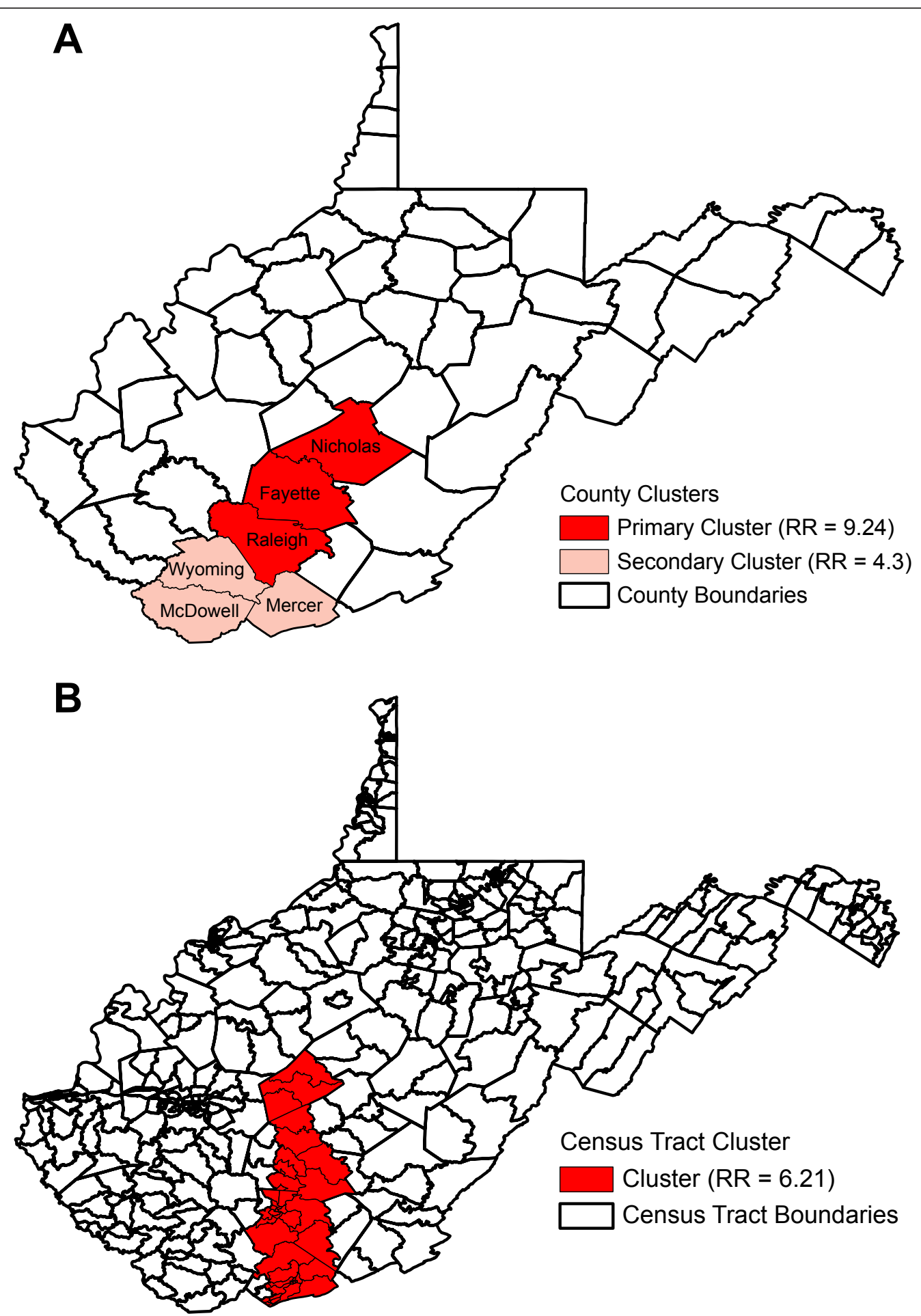

Figure 2 Spatial clustering of La Crosse virus infection risk at the county and census tract levels in children 15 years and younger. These maps show the significant high-risk clusters for La Crosse virus infection in West Virginia at the county (A) and at the census tract levels (B) detected by Kulldorff's Spatial Scan Statistic. RR = relative risk. 
Table 2 Characteristics of Reported La Crosse Virus Infection Cases in West Virginia, 2003 to 2007

\begin{tabular}{lc}
\hline Variable & Number (\%) \\
\hline Sex & \\
Male & $58(60.4)$ \\
Female & $38(39.6)$ \\
Age & \\
$0.4-0.9$ yrs & $1(1.0)$ \\
$1-5$ yrs & $27(28.1)$ \\
$6-10$ yrs & $38(39.6)$ \\
$11-15$ yrs & $15(15.6)$ \\
16- 20 yrs & $6(6.3)$ \\
$\geq 21$ yrs & $9(9.4)$ \\
Month of presentation & \\
May & $1(1.0)$ \\
June & $8(8.3)$ \\
July & $27(28.1)$ \\
August & $36(37.5)$ \\
September & $16(16.6)$ \\
October & $8(8.3)$ \\
Reported Signs and Symptoms & \\
Fever & $73(76.0)$ \\
Vomiting & $72(75.0)$ \\
Elevated CSF WBC & $68(70.8)$ \\
Photophobia & $50(52.1)$ \\
Nausea & $48(50.0)$ \\
Weakness & $40(41.6)$ \\
Meningitis & $39(40.6)$ \\
Encephalitis & $38(39.6)$ \\
Stiff Neck & $32(33.3)$ \\
Confusion & $32(33.3)$ \\
Seizures & $23(24.0)$ \\
Elevated CSF protein & $24(25.0)$ \\
Myalgia & $11(11.5)$ \\
\hline & $7(7.3)$ \\
Rash & $7(7.3)$ \\
Comalgia & $4(4.2)$ \\
& \\
\hline
\end{tabular}

(24.0\%), and coma (4.2\%). Meningitis and encephalitis were reported in $41.6 \%$ and $40.6 \%$ of the cases, respectively. A CFR of $3.1 \%$ was observed.

Health care providers reported either the presence of elevated cerebral spinal fluid (CSF) white blood cell (WBC) counts and/or the determined the counts. Elevated CSF WBC counts were reported in 73 cases (76.0\%), of which 68 cases had a mean CSF WBC count of 160.9 per $\mathrm{mm}^{3}$ (range: 10 to 670 per $\mathrm{mm}^{3}$, SD: 153.8 per $\mathrm{mm}^{3}$ ). Health care providers also either reported the presence of elevated CSF protein levels and/or the CSF protein levels. Elevated CSF protein levels were reported
Table 3 The Observed and Reported Environmental Conditions Present at the Primary Residences of La Crosse Virus Infection Cases in West Virginia, 2003 to 2007

\begin{tabular}{|c|c|c|}
\hline Variable & Present (\%) & Missing Data \\
\hline Containers* & $60(70.6)$ & 11 \\
\hline Tires & $33(40.7)$ & 15 \\
\hline Tarps & $32(41.6)$ & 19 \\
\hline Other containers $^{\dagger}$ & $53(63.1)$ & 12 \\
\hline Standing water & $41(49.4)$ & 13 \\
\hline Wooded area & $81(92.0)$ & 8 \\
\hline Hardwood & $68(94.4)$ & 24 \\
\hline Evergreen & $35(71.4)$ & 47 \\
\hline
\end{tabular}

${ }^{*}$ Containers were all containers including: other containers, tires, and tarps. These variables were cross-referenced with one another for each case to determine the presence of at least one variable at each case site.

${ }^{\dagger}$ Other containers were those containers not including tires and/or tarps.

in 24 cases $(25.0 \%)$. Cerebral spinal fluid protein levels were only available for 27 cases, with a mean level of 64.4, mg/dl (range: 24 to $359 \mathrm{mg} / \mathrm{dl}, \mathrm{SD}: 61.6 \mathrm{mg} / \mathrm{dl}$ ). Fever was present in all cases for which temperature was reported $(\mathrm{n}=54)$, with a mean value of $39.4^{\circ} \mathrm{C}$ (range: $38.3^{\circ} \mathrm{C}$ to $40.6^{\circ} \mathrm{C}$, SD: $0.66^{\circ} \mathrm{C}$ ).

\section{Environmental observations}

The most commonly observed environmental variables at case residences were the presence of a wooded area $(92.0 \%)$ and the presence of containers (70.5\%), which provide suitable habitats for mosquito larval development (Table 3). The presence of standing water was observed at almost half of the case residences (49.4\%) and the majority of case residences (76.7\%) were located within $45.6 \mathrm{~m}(149.0 \mathrm{ft})$ of a wooded area (Table 4$)$.

\section{Discussion}

Traditionally the highest number of reported LACV infection cases came from the upper-Midwestern states, but during the last 20 years the geographic area with the highest number of reported cases shifted to the Appalachian region of the United States [8]. Within this region, West Virginia now leads the nation in the number of reported cases annually [8]. To investigate the epidemiology of LACV infections in West Virginia, we performed spatial and descriptive analyses of LACV infection cases reported to the West Virginia Department of Health and Human Resources from 2003 to 2007.

The results of this study found that the highest cumulative incidences of LACV infection were located in the south-central region of the state, similar to the results of previous studies [27,28]. Furthermore, both the unsmoothed and SEB smoothed cumulative incidences were the highest in this region at both the county and 


\begin{tabular}{|c|c|c|}
\hline Variable & Number (\%) & Missing Data \\
\hline No. of tires & & 20 \\
\hline 0 & $49(64.5)$ & \\
\hline $1-9$ & 21 (27.6) & \\
\hline$\geq 10$ & $7(9.2)$ & \\
\hline No. of tarps & & 31 \\
\hline 0 & $46(70.8)$ & \\
\hline $1-10$ & $21(32.3)$ & \\
\hline $\begin{array}{l}\text { No. of other types of } \\
\text { Containers* }\end{array}$ & & 23 \\
\hline 0 & $19(26.0)$ & \\
\hline $1-5$ & $29(39.7)$ & \\
\hline $6-15$ & $10(13.7)$ & \\
\hline$\geq 16$ & $3(4.1)$ & \\
\hline $\begin{array}{l}\text { Distance (meters) from } \\
\text { residence to wooded } \\
\text { area }\end{array}$ & & 23 \\
\hline $0-14.9(0-49 \mathrm{ft})$ & $35(47.9)$ & \\
\hline $15-45.6(50-149 \mathrm{ft})$ & $21(28.8)$ & \\
\hline$\geq 45.7(\geq 150 \mathrm{ft})$ & $6(8.2)$ & \\
\hline
\end{tabular}

*Other types of containers were those containers not including tires and/or tarps.

census tract levels. To determine those areas at the highest risk for disease, we performed spatial analyses at two different spatial levels (county and census tract). The census tract level was used in this study over a smaller geographic level (i.e., census block) because this is typically the smallest spatial level that is acceptable for the public reporting of diseases under the Health Insurance Portability and Accountability Act (HIPAA) and due to the availability of census data variables during the study period. The high cumulative incidences observed in this study were similar to those high cumulative incidences reported in eastern Tennessee [36], the location of another area of high-risk LACV infection case clustering in the Appalachian region [8,36]. Significant high-risk clustering was observed at both the county and census tract levels in the south-central region of West Virginia. Two statistically significant clusters of high LACV infection risk (comprising six counties) were detected at the county level with a relative risk roughly four to nine fold higher than those counties located outside of the high-risk clusters, and one statistically significant cluster (comprising 41 census tracts within six counties) was observed at the census tract level with a relative risk about six times higher compared to those census tracts located outside of the high-risk cluster. These areas of high-risk should form the focus of education and intervention efforts in the future. Additionally, the results of this study reaffirm that the census tract level is the preferred geographic level for reporting cases and conducting analyses of focal diseases $[36,41]$.

In agreement with previous work $[7,8]$, our study found that the majority of reported LACV infections occur in male children 15 years and younger during the summer months, and that cases display fever, headache, vomiting, and mental status changes. La Crosse virus infection has traditionally proven difficult to distinguish from herpes simplex meningoencephalitis [7,42]. Our study demonstrates that elevated CSF WBCs remain a hallmark of LACV infection, however it should be noted that patients frequently demonstrate a predominance of polymorphonuclear leukocytes on their peripheral blood smears and in their CSF, which is suggestive of a bacterial infection and may result in a delayed diagnosis of LACV infection [7]. A troubling discovery in this study was the continued high CFR (3.1\%) in West Virginia [28], compared to a lower CFR (1.5\%) reported during the same time period for the rest of the United States [8]. The reason for this higher CFR in West Virginia is unclear, but may indicate the possibility of a more virulent strain(s) of the virus circulating in this region $[43,44]$, reporting bias, clinical management, and/or chance variation. The reasons underlying the increased CFR warrant further investigation.

The reason for the increase in the number of reported cases of LACV infection in the Appalachia region and in West Virginia, in the past 20 years remains unknown. One possibility is a change in the geographic distribution of vector species, and recent work has indicated a possible geographic shift in vector abundance between Ae. triseriatus, Ae. albopictus, and Ae. japonicus populations in West Virginia [45-47]. Of interest, the number of reported cases of LACV infection in West Virginia has decreased in recent years (2005 to 2009) when compared to data from the previous decade (CDC unpublished). Prior to the establishment of Ae. albopictus and Ae. japonicus in West Virginia, LACV had been isolated from Ae. triseriatus mosquitoes collected in 1996 at former sites of confirmed LACV infection cases in West Virginia [26]. A recent survey of abandoned tires to determine the presence of mosquito larvae in western, central and eastern West Virginia found that Ae. japonicus was the most frequently collected larval mosquito species throughout the state in this habitat type. Furthermore, their survey revealed that Ae. albopictus was collected at significantly lower numbers than Ae. triseriatus at both peridomestic and non-peridomestic tire sites in the central region of the state [45], where the results of this study indicate the highest disease risk. However, a study in eastern Tennessee collecting adult host-seeking mosquitoes using $\mathrm{CO}_{2}$ baited $\mathrm{CDC}$ traps from 2004 to 2006 found that Ae. albopictus was three times more likely to be collected 
than Ae. triseriatus at the residences of previously confirmed LACV cases, in addition to residences without a prior history of reported LACV cases [14]. Similar results were observed at active LACV case residences using human landing collections in 2004 [14].

The observation that a large number of case residences in this study were in close proximity to wooded areas and had the presence of containers are in agreement with those findings of a case-control study previously performed in this region which found them to be risk factors for LACV infection in West Virginia [28]. Such characteristics provide a habitat for vector species and amplification hosts [20-24]. Thus, as observed elsewhere, the closure of tree-holes and the elimination of water-holding containers would likely reduce the overall burden of infective mosquitoes and decrease the risk of LACV transmission [48]. Importantly, although LACV has not been isolated from mosquitoes in West Virginia since 1996, the lack of isolates are likely the result of extremely limited testing of mosquito pools for LACV rather than a true lack of virus presence in mosquito populations. Ultimately, the status of Ae. triseriatus, Ae. albopictus, and Ae. japonicus concerning the transmission and maintenance of LACV in West Virginia remains unknown, and research is urgently needed to fill this gap in our understanding of the epidemiology of LACV.

\section{Limitations}

Due to the methodology and data employed in our study there are some limitations. Passive surveillance systems are prone to under-reporting/detection of disease, though we feel that the majority of cases suffering from severe illness were diagnosed and reported to state health officials. Clinical data were collected from multiple health care providers by local health department personnel for the purpose of case ascertainment. Due to limited resources, verification of complete and accurate reporting of laboratory and clinical features was not possible. As data regarding the clinical features of cases of LACV infection were extracted from patient charts and/or recalled by healthcare professionals post-presentation of disease, the actual frequency of reported signs and symptoms could be higher than those reported in this study. Missing information for environmental data resulted when visits to the cases' residences were not possible. The environmental information presented in this paper is purely for descriptive purposes and is not intended to infer an association with LACV infections.

\section{Conclusions}

During the study period, West Virginia reported the highest cumulative incidence of LACV infection cases in the United States, marking a shift from the traditional geographic focus of reported LACV infection cases from the upper-Midwestern states to the Appalachian region [8]. The reason for this shift is unclear, but could be due to a variety of factors including increases and/or decreases in diagnosis, reporting, education, and prevention. Additionally, changes in virus strain virulence, the distribution of vector species, land use patterns, forest succession and/or increased human encroachment into enzootic foci could also be contributing factors. Future surveillance efforts should include both the screening of vector species for virus, as well as determining the seroprevalence to LACV in amplification hosts. In addition, LACV isolations are needed so West Virginian virus strains can be compared with virus strains from other regions to determine if differences exist in virulence and vector competence. Although the reporting of the clinical presentation of cases of LACV infection through a passive surveillance system is not without limitations, such reporting will allow for the continued monitoring of infection patterns. The findings of this study demonstrate that south-central West Virginia remains a focus of LACV transmission and highlights the utility of using the combination of cumulative incidence, relative risk, and spatial statistics to determine those geographic areas at highest risk for infection. The majority of reported LACV infection cases during the study period had the presence of wooded areas and containers near their primary residence corroborating the results of a previous case-control study. Finally, the results of this study will be useful for guiding disease control strategies and will allow public health officials to target specific areas for interventions.

\section{Acknowledgements}

We would like to offer our sincere thanks to the staff of the many local health departments in West Virginia who collected La Crosse virus infection case data and submitted them to the West Virginia Department of Health and Human Resources. Without their contributions this work would not have been possible. This study was partially supported by the John S. Dunn Fund through the University of Texas Medical Branch (ADH).

\section{Author details}

${ }^{1}$ The University of Tennessee, Department of Entomology \& Plant Pathology, Knoxville, TN 37996-4560, USA. ${ }^{2}$ The University of Texas Medical Branch, Department of Pathology and the Center for Biodefense and Emerging Infectious Diseases, 301 University Blvd. Galveston, Texas 77555-0609, USA. ${ }^{3}$ West Virginia Bureau for Public Health, Room 125, 350 Capitol St. Charleston, WV 25301, USA. ${ }^{4}$ The University of Tennessee, Department of Comparative Medicine, College of Veterinary Medicine 2407 River Dr. Knoxville, TN 37996-4543, USA.

\section{Authors' contributions}

$\mathrm{ADH}$ conceived and designed the study, performed the experiments, and wrote the manuscript. DB contributed the data and participated in helping draft the manuscript. AO helped design the study, perform the experiments, and participated in helping draft the manuscript. All authors read and approved the final manuscript.

\section{Competing interests}

The authors declare that they have no competing interests.

Received: 18 March 2010 Accepted: 26 January 2011

Published: 26 January 2011 


\section{References}

1. Thompson WH, Kalfayan B, Anslow RO: Isolation of California encephalitis group virus from a fatal human illness. Am J Epidemiol 1965, 81:245-253.

2. Calisher $\mathrm{CH}$ : Medically important arboviruses of the United States and Canada. Clin Microbiol Rev 1994, 7(1):89-116.

3. Tsai TF: Arboviral infections in the United States. Infect Dis Clin North Am 1991, 5:73-102.

4. Grimstad P: California group virus disease. In In: The arboviruses: epidemiology and ecology. Volume II. Edited by: Monath TP. Boca Raton, FL: CRC Press; 1988:99-136

5. Kappus KD, Monath TP, Kaminski RM, Calisher CH: Reported encephalitis associated with California serogroup virus infections in the United States, 1963-1981. Prog Clin Biol Res 1983, 123:31-41.

6. McJunkin JE, Khan RR, Tsai TF: California-La Crosse encephalitis. Infect Dis Clin North Am 1998, 12(1):83-93.

7. Rust RS, Thompson WH, Matthews CG, Beaty BJ, Chun RW: La Crosse and other forms of California encephalitis. J Child Neurol 1999, 14(1):1-14.

8. Haddow AD, Odoi A: The incidence risk, clustering, and clinical presentation of La Crosse virus infections in the eastern United States, 2003-2007. PLoS One 2009, 4(7):e6145.

9. Jones TF, Craig AS, Nasci RS, Patterson LE, Erwin PC, Gerhardt RR, Ussery XT, Schaffner W: Newly recognized focus of La Crosse encephalitis in Tennessee. Clin Infect Dis 1999, 28(1):93-97.

10. Watts DM, Morris CD, Wright RE, DeFoliart GR, Hanson RP: Transmission of La Crosse virus (California encephalitis group) by the mosquito Aedes triseriatus. J Med Entomol 1972, 9(2):125-127

11. Gerhardt RR, Gottfried KL, Apperson CS, Davis BS, Erwin PC, Smith AB Panella NA, Powell EE, Nasci RS: First isolation of La Crosse virus from naturally infected Aedes albopictus. Emerg Infect Dis 2001, 7(5):807-811.

12. Lambert AJ, Blair CD, D'Anton M, Ewing W, Harborth M, Seiferth R, Xiang J, Lanciotti RS: La Crosse virus in Aedes albopictus mosquitoes, Texas, USA Emerg Infect Dis 2009, 16(5):856-858.

13. Sardelis MR, Turell MJ, Andre RG: Laboratory transmission of La Crosse virus by Ochlerotatus j. japonicus (Diptera: Culicidae). J Med Entomol 2002, 39(4):635-639.

14. Haddow AD, Gerhardt RR, Jones CJ, Odoi A: The mosquitoes of eastern Tennessee: studies on abundance, habitat preferences, and host-seeking behaviors. J Vector Ecol 2009, 34:70-80.

15. Molaei G, Farajollahi A, Scott JJ, Gaugler R, Andreadis TG: Human bloodfeeding by the recently introduced mosquito, Aedes japonicus japonicus, and public health implications. J Am Mosq Control Assoc 2009, 25(2):210-214.

16. Niebylski ML, Savage HM, Nasci RS, Craig GB Jr: Blood hosts of Aedes albopictus in the United States. J Am Mosq Control Assoc 1994, 10(3):447-450.

17. Watts DM, Pantuwatana S, DeFoliart GR, Yuill TM, Thompson WH: Transovarial transmission of La Crosse virus (California encephalitis group) in the mosquito, Aedes triseriatus. Science 1973, 182(117):1140-1141.

18. Watts DM, Grimstad PR, DeFoliart GR, Yuill TM, Hanson RP: Laboratory transmission of La Crosse encephalitis virus by several species of mosquitoes. J Med Entomol 1973, 10:583-586.

19. Watts DM, Thompson WH, Yuill TM, DeFoliart GR, Hanson RP: Overwintering of La Crosse virus in Aedes triseriatus. Am J Trop Med Hyg 1974, 23(4):694-700

20. Moulton DW, Thompson WH: California group virus infections in small, forest-dwelling mammals of Wisconsin. Some ecological considerations. Am J Trop Med Hyg 1971, 20(3):474-482.

21. Ksiazek TG, Yuill TM: Viremia and antibody response to La Crosse virus in sentinel gray squirrels (Sciuris carolinensis) and chipmunks (Tamias striatus). Am J Trop Med Hyg 1977, 26(4):815-821

22. Thompson WH, Evans AS: California encephalitis virus studies in Wisconsin. Am J Epidemiol 1965, 81:230-244

23. Kitron U, Michael J, Swanson J, Haramis L: Spatial analysis of the distribution of La Crosse encephalitis in Illinois, using a geographic information system and local and global spatial statistics. Am J Trop Med Hyg 1997, 57(4):469-475.

24. Erwin PC, Jones TF, Gerhardt RR, Halford SK, Smith AB, Patterson LE, Gottfried KL, Burkhalter KL, Nasci RS, Schaffner W: La Crosse encephalitis in Eastern Tennessee: clinical, environmental, and entomological characteristics from a blinded cohort study. Am J Epidemiol 2002, 155(11):1060-1065
25. McJunkin JE, de los Reyes EC, Irazuzta JE, Caceres MJ, Khan RR, Minnich LL, Fu KD, Lovett GD, Tsai T, Thompson A: La Crosse encephalitis in children. N Engl J Med 2001, 344(11):801-807.

26. Nasci RS, Moore CG, Biggerstaff BJ, Panella NA, Liu HQ, Karabatsos N, Davis BS, Brannon ES: La Crosse encephalitis virus habitat associations in Nicholas County, West Virginia. J Med Entomol 2000, 37(4):559-570.

27. La Crosse encephalitis in West Virginia. MMWR Morb Mortal Wkly Rep 1988, 37(5):79-82.

28. Woodruff BA, Baron RC, Tsai TF: Symptomatic La Crosse virus infections of the central nervous system: a study of risk factors in an endemic area. Am J Epidemiol 1992, 136(3):320-327.

29. CDC: Neuroinvasive and non-neuroinvasive domestic arboviral diseases: 2004 case definition. In National Center for Public Health Informatics (NCPHI). 2004 edition. Edited by: NNDSS. Atlanta, Georgia: Centers for Disease Control; 2004

30. US Census Bureau: Cartographic Boundary File Web Site. 2008 [http:// www.census.gov/geo/www/tiger/index.html], Accessed August 32009.

31. STATA: Intercooled STATA Version 10.0 for Macintosh. College Station, Texas, USA: STATA Corporation; 2007.

32. Clayton D, Bernardinelli L: Bayesian methods for mapping disease risk. Oxford: Oxford University Press; 1997.

33. Odoi A, Martin SW, Michel P, Holt J, Middleton D, Wilson J: Geographical and temporal distribution of human giardiasis in Ontario, Canada. Int $J$ Health Geogr 2003, 2(1):5

34. Bernardinelli L, Montomoli C: Empirical Bayes versus fully Bayesian analysis of geographical variation in disease risk. Stat Med 1992, 11:983-1007.

35. Bithell JF: A classification of disease mapping methods. Stat Med 2000, 19:2203-2215.

36. Haddow $A D$, Jones $C J$, Odoi $A$ : Assessing risk in focal arboviral infections: Are we missing the big or little Picture? PLoS One 2009, 4(9):e6954.

37. Anselin L: GeoDa Version 0.95i. Spatial Analysis Lab, University of Illinois Urbana-Champaign, IL, USA; 2003

38. Kulldorff M: A spatial scan statistic. Commun Stat Theory Methods 1997, 26:1481-1496.

39. Kulldorff M: SaTScan Version 9.0.1. Software for the spatial and space-time scan statistics Information Management Services, Boston, MA, USA; 2010

40. ESRI: ArcView GIS Version 9.2. Environmental Systems Research Institute, Inc., Redlands, CA, USA; 2006.

41. Winters AM, Eisen RJ, Delorey MJ, Fischer M, Nasci RS, Zielinski-Gutierrez E, Moore CG, Pape WJ, Eisen L: Spatial risk assessments based on vectorborne disease epidemiologic data: importance of scale for West Nile virus disease in Colorado. Am J Trop Med Hyg 82(5):945-953.

42. Sokol DK, Kleiman MB, Garg BP: La Crosse viral encephalitis mimics herpes simplex viral encephalitis. Pediatr Neurol 2001, 25(5):413-415.

43. Huang C, Thompson WH, Karabatsos N, Grady L, Campbell WP: Evidence that fatal human infections with La Crosse virus may be associated with a narrow range of genotypes. Virus Res 1997, 48(2):143-148.

44. Reese SM, Blitvich BJ, Blair CD, Geske D, Beaty BJ, Black WCt: Potential for La Crosse virus segment reassortment in nature. Virol J 2008, 5:164.

45. Joy JE, Sullivan SN: Occurrence of tire inhabiting mosquito larvae in different geographic regions of West Virginia. J Am Mosq Control Assoc 2005, 21(4):380-386

46. Joy JE: Larval mosquitoes in abandoned tire pile sites from West Virginia. J Am Mosa Control Assoc 2004, 20(1):12-17.

47. Joy JE, Hanna AA, Kennedy BA: Spatial and temporal variation in the mosquitoes (Diptera: Culicidae) inhabiting waste tires in Nicholas County, West Virginia. J Med Entomol 2003, 40(1):73-77.

48. Parry JE: Control of Aedes triseriatus in La Crosse, Wisconsin. Prog Clin Biol Res 1983, 123.355-363.

Pre-publication history

The pre-publication history for this paper can be accessed here: http://www.biomedcentral.com/1471-2334/11/29/prepub

doi:10.1186/1471-2334-11-29

Cite this article as: Haddow et al.: The spatial epidemiology and clinical features of reported cases of La Crosse Virus infection in West Virginia from 2003 to 2007. BMC Infectious Diseases 2011 11:29. 